\title{
AURC All Normalized by Body Mass Index
}

National Cancer Institute

\section{Source}

National Cancer Institute. AURC All Normalized by Body Mass Index. NCI Thesaurus.

Code C92342.

The area under the urinary excretion rate curve (AURC) from time zero to the last measurable rate divided by the body mass index. 